\title{
Effect of Pharmacological Mydriasis on the
} Intraocular Pressure in Eyes with Filtering Blebs Compared to Normal Eyes: A Pilot Study

\author{
Hani M Gharieb Ibrahim (D) \\ Ophthalmology Department, Ain Shams University, Cairo, Egypt \\ Correspondence: Hani M Gharieb Ibrahim, Ophthalmology Department, Ain Shams University Faculty of Medicine, Ramsees Street, Abbaseya, Cairo, \\ Egypt, Email hani.gharieb@med.asu.edu.eg; extrahany@yahoo.com
}

\begin{abstract}
Aim: To test the effect of pharmacological mydriasis on intraocular pressure (IOP) in eyes with functioning blebs compared with normal eyes.

Patients and Methods: The cross-sectional study included 39 eyes, of which 19 eyes had filtering trabeculectomy blebs (group A), and 20 eyes with no previous surgeries (group B). IOP was measured using the Goldmann applanation tonometer before, and 30 min after the application of tropicamide $1 \%$ eye drops.

Results: In group A eyes, the IOP level changed from $13.68 \pm 3.4 \mathrm{mmHg}$ before pupillary dilatation, to achieve a level of $11.95 \pm 3.03$ $\mathrm{mmHg}$ following mydriasis, with an average IOP drop of $1.73 \mathrm{mmHg}(\mathrm{p}<0.0001)$. On the other hand, the pre-dilatation IOP in group B eyes was $15.9 \pm 3.00 \mathrm{mmHg}$, had increased after mydriasis to $18.4 \pm 2.9 \mathrm{mmHg}$, with an average rise of $2.5 \mathrm{mmHg}(\mathrm{p}<0.0001)$. The difference in IOP change following mydriasis between both groups was significant $(\mathrm{p}<0.0001)$.

Conclusion: Surprisingly, pharmacological pupillary dilatation resulted in a reduction of IOP in eyes with successful trabeculectomy, which is different from the usual effect of raising the IOP as in the control group. This effect has not been previously studied.
\end{abstract}

Keywords: mydriasis, intraocular pressure, trabeculectomy, filtering bleb

\section{Introduction}

Mydriatic eye drops are frequently used in Ophthalmology outpatient clinics for the diagnosis of posterior segment and lens disorders. Tropicamide is an anticholinergic drug frequently used for dilating the pupil. Tropicamide acts within 20 minutes and its duration of action is approximately 4-8 hours. ${ }^{1}$ An elevation in IOP level has been observed after pharmacological mydriasis. ${ }^{2}$ This IOP rise following pupillary dilatation may be attributed to the reduction in aqueous outflow secondary to the decreased traction on the trabecular meshwork resulting from ciliary muscle paralysis. ${ }^{3}$ Moreover, iris pigment dispersion in the anterior chamber can cause obstruction of the trabecular meshwork representing a possible mechanism of IOP rise after mydriasis. ${ }^{4}$

It is known that narrow angle is a predisposing factor for acute IOP elevation following pupil dilatation; however, IOP elevation has also been found to occur following mydriasis in eyes that do not have narrow angles. ${ }^{5}$

The current study aimed to study the changes in IOP following mydriasis in eyes with filtering blebs compared to non-operated eyes.

\section{Patients and Methods}

This cross-sectional pilot study, held in a clinical practice, included 39 eyes of patients attending the Ophthalmology outpatient clinic in the university hospital with various complaints that required pupil dilation for lens and fundus examination. This study adhered to tenets of the Declaration of Helsinki. This study was approved by the ethical committee of the Ain Shams university. Informed consent was obtained from all the patients. The examinations performed represent part of the general 
ophthalmic examination done at the ophthalmology outpatient clinics, which carried no additional risks for the patients. The eyes were divided into the following two groups:

Group A: included 19 eyes of with filtering blebs following successful trabeculectomy. Trabeculectomy was performed in these eyes previously for primary open-angle glaucoma (POAG) non-responding to maximum topical therapy.

Group B: This group included 20 eyes that had no previous surgeries.

Exclusion criteria included eyes with closed or narrow angles, eyes with poorly dilating pupils, eyes with failed trabeculectomy blebs (flat or encapsulated blebs associated with high IOP), eyes using antiglaucoma drops, as well as eyes with anterior segment pathology such as corneal scars, edema, or thinning.

Gonioscopy was performed using the Goldmann three-mirror contact lens (Haag-Streit Diagnostics, UK), which was performed under topical anaesthesia and methylcellulose was used as a coupling agent for the lens. The aim of gonioscopy was to determine the angle width (according to the Shaffer system), ${ }^{6}$ and to check the patency of the trabeculectomy internal ostium. Only eyes with wide-angle grades 3 or 4 were included. Eyes with narrow angles as well as eyes with occluded trabeculectomy internal ostium were excluded.

Then, IOP was measured using the Goldmann applanation tonometer (Haag-Streit Diagnostics, UK). Topical anaesthetic benoxinate $0.4 \%$ was applied first, followed by the application of a fluorescein strip in the lower conjunctival sac for 3 seconds, then the IOP was measured using a well-calibrated Goldmann applanation tonometer, by an experienced Ophthalmology consultant. The tonometer knob was masked during IOP examination, unmasking was done after commencing the IOP measurement to reduce possible bias. IOP measurement was performed once before pupil dilatation. Tropicamide $1 \%$ eye drops were applied in the lower conjunctival fornix twice, 10 min apart. IOP measurement was repeated 30 min following the application of the first dose of tropicamide after checking the pupil diameter to confirm full mydriasis.

Furthermore, slit lamp examination was performed for anterior segment examination, in addition to examining the bleb to determine bleb extent, elevation, vascularity, and Seidel test.

In group A, bleb morphology was assessed according to the Indiana Bleb Appearance Grading Scale. ${ }^{7}$ Blebs included in the current study had at least moderate $(\mathrm{H} 2)$ or high $(\mathrm{H} 3)$ bleb elevation, horizontal extent of at least 1 clock hour (E1 at least), avascular or have mild vascularity (maximum V2), with no bleb leak. Flat blebs and encapsulated blebs (Tenon's cysts) were excluded.

All examinations were performed gently to avoid eye compression.

Data were recorded and subjected to statistical analysis using the $t$-test for comparison of means, Pearson's correlations, and linear regression analysis, using MedCalc version 20.006 (C) 2021, MedCalc Software Ltd, Ostend, Belgium). Differences were considered significant at $\mathrm{p} \leq 0.05$.

\section{Results}

The average age of the patients in group A was $67.1 \pm 13.4$ years, the group included ten males and nine females. Group $\mathrm{B}$ included ten males and ten females, with an average age of $60.4 \pm 5$ years. Group A included 9 eyes with mild hyperopia ( $\leq 2.00 \mathrm{D}$ ), 4 eyes with mild myopia (up to $-2.00 \mathrm{D}$ ), and 6 emmetropic eyes. While group B included 7 eyes with mild hyperopia, 6 eyes with mild myopia, and 7 emmetropic eyes.

In group A, the IOP level measured before mydriasis was $13.68 \pm 3.4 \mathrm{mmHg}$, and after full mydriasis, the IOP was $11.95 \pm 3.03 \mathrm{mmHg}$, with about $1.73 \mathrm{mmHg}$ drop of IOP $(\mathrm{P}<0.0001)$. IOP level reduction following mydriasis was the case in 15 eyes (78.9\%), the amplitude of IOP drop ranged from 1 to $4 \mathrm{mmHg}$, while the IOP remained the same in 4 eyes (21.1\%). On the other hand, the pre-dilatation IOP in group B was $15.9 \pm 3.00 \mathrm{mmHg}$, while the IOP level was $18.4 \pm 2.9 \mathrm{mmHg}$ following pupillary dilatation, with an average rise of $2.5 \mathrm{mmHg}(\mathrm{P}<0.0001)$. The rise of IOP following mydriasis in this group occurred in 19 eyes (95\%), the magnitude of IOP rise was $1 \mathrm{mmHg}$ in one eye, while in other eyes it rose $2-4 \mathrm{mmHg}$, IOP remained the same following pupil dilatation in one eye (5\%). There was a significant difference in pre-dilatation IOP between the two groups $(\mathrm{P}<0.05)$. IOP levels are shown in Figure 1.

The difference in IOP change following mydriasis between the two groups was significant $(\mathrm{P}<0.0001)$. 


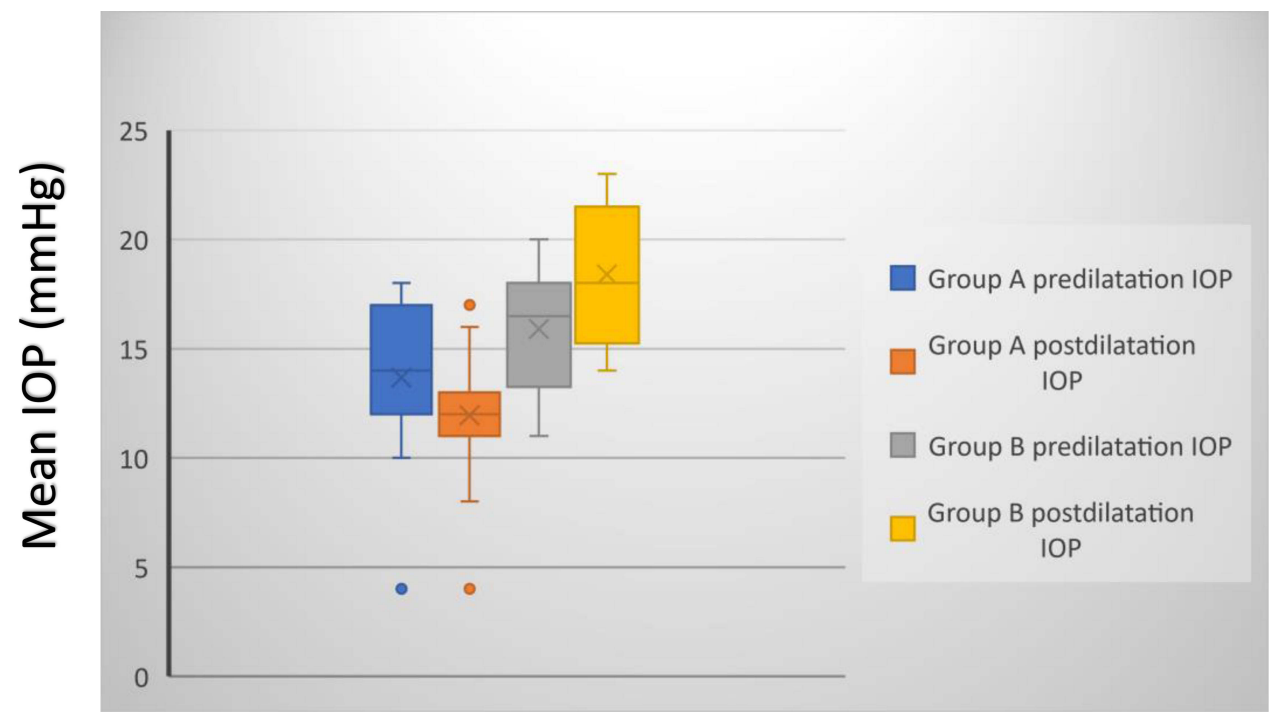

Figure I Pre-dilatation and post-dilatation IOP levels in group A and group B eyes.

Moreover, gonioscopy performed using the Goldmann three-mirror gonio lens showed open anterior chamber angles (grades 3 and 4) in all examined eyes. In group A, gonioscopy revealed a patent trabeculectomy internal ostium in all eyes.

Slit lamp examination showed successful trabeculectomy with filtering blebs in group A (according to the Indiana bleb grading scale as described before), while in group B, there was no evidence of previous ocular surgery. There were no anterior segment pathologies in any of the eyes.

There was a negative correlation between pre-dilatation IOP and the difference in IOP (pre-dilatation - postdilatation) in group A $(\mathrm{r}=-0.4491, \mathrm{P}=0.0537)$. Moreover, a strong correlation was found between pre-dilatation and post-dilatation IOP levels in group A $(\mathrm{r}=0.9149, \mathrm{P}<0.0001)$. Similarly, pre-dilatation and post-dilatation IOP levels in group B were strongly correlated $(\mathrm{r}=0.9376, \mathrm{P}<0.0001)$. A less significant correlation was found between pre-dilatation IOP and the difference in IOP (pre-dilatation - post-dilatation) in group $\mathrm{B}(\mathrm{r}=-0.2497, \mathrm{P}=0.2883)$.

Figures 2 and 3 show the results of linear regression analysis. The correlations between the pre-dilatation IOP and the difference in IOP (pre-dilatation - post-dilatation) in both groups are shown.

\section{Discussion}

Pharmacological pupillary dilatation is known to cause an IOP elevation. The current study showed that this was not the case in eyes with a filtering trabeculectomy bleb, where these eyes attained lower IOP level following pharmacological mydriasis, a novel finding which has not been addressed in any previous study. On the other hand, eyes with no previous surgeries (group B) had an IOP rise following pupillary dilatation in a similar manner to the outcome of previous studies.

Many studies had shown that pharmacological pupillary dilatation is associated with an increase in IOP. This IOP rise was attributed to reduced aqueous drainage due to decreased traction on the trabecular meshwork, secondary to ciliary muscle paralysis. ${ }^{3}$

For example, in one study, there was a significant increase in IOP following pharmacological mydriasis. ${ }^{8}$ Similarly, significant IOP elevation occurred in $32 \%$ of patients with open-angle glaucoma following pupil dilatation with $2.5 \%$ phenylephrine and $1 \%$ tropicamide in another study. ${ }^{9}$ Other studies reported comparable rise of IOP following pupil dilatation. ${ }^{3,10-12}$ These findings were comparable to the results of our study group B, in which IOP achieved higher level following mydriasis.

The trabecular meshwork is the site of aqueous outflow resistance in normal eyes and in POAG. However, in eyes with successful trabeculectomy, the scleral flap and the episcleral tissue determine the resistance to aqueous outflow. ${ }^{13}$ So, the usual resistance to aqueous outflow at the trabecular meshwork has been bypassed by the trabeculectomy, which could explain the lack of IOP rise following mydriasis in the current study group A. 


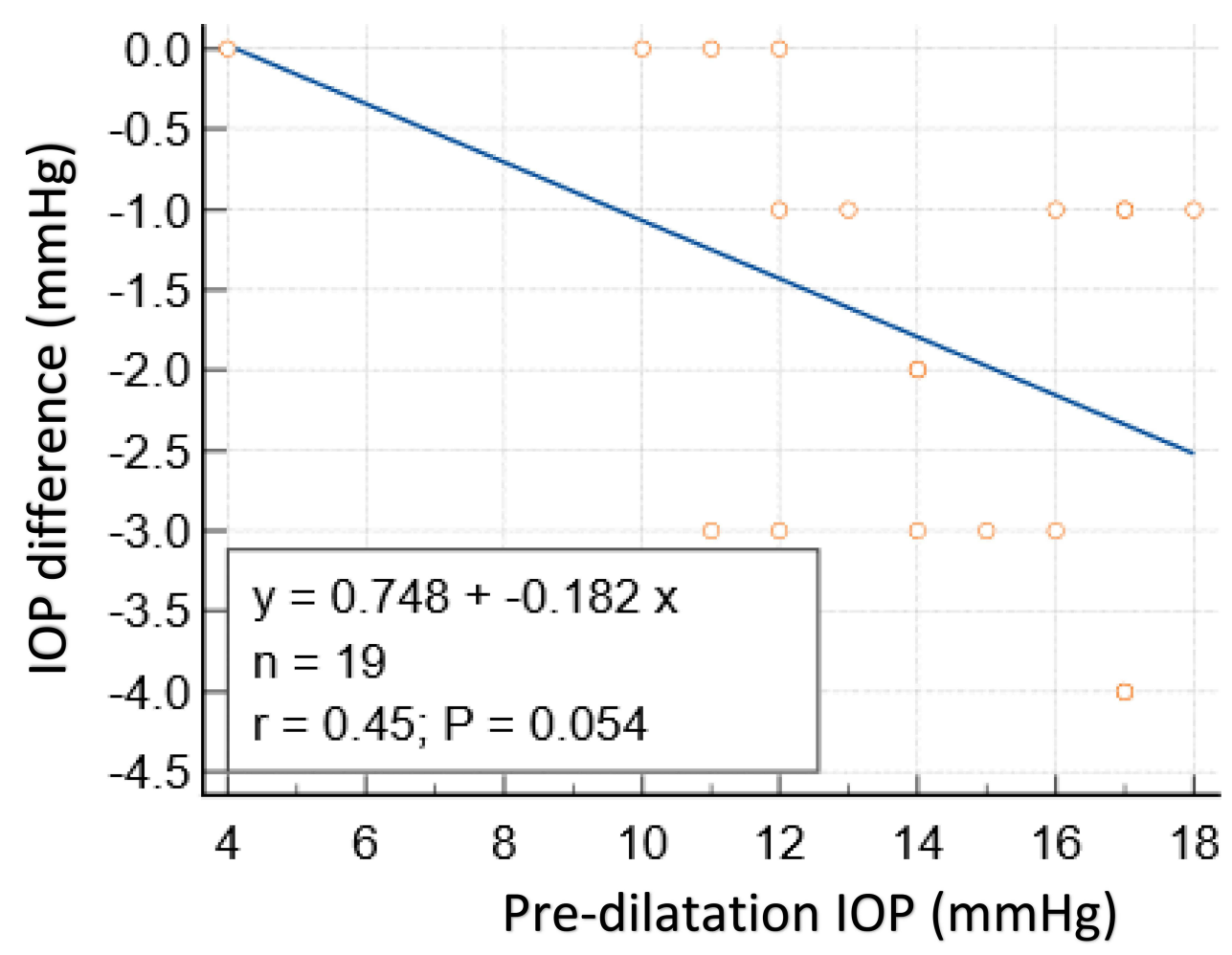

Figure 2 Linear regression analysis showing the correlation between the difference in IOP (pre-dilatation - post-dilatation) and the pre-dilatation IOP in group A.

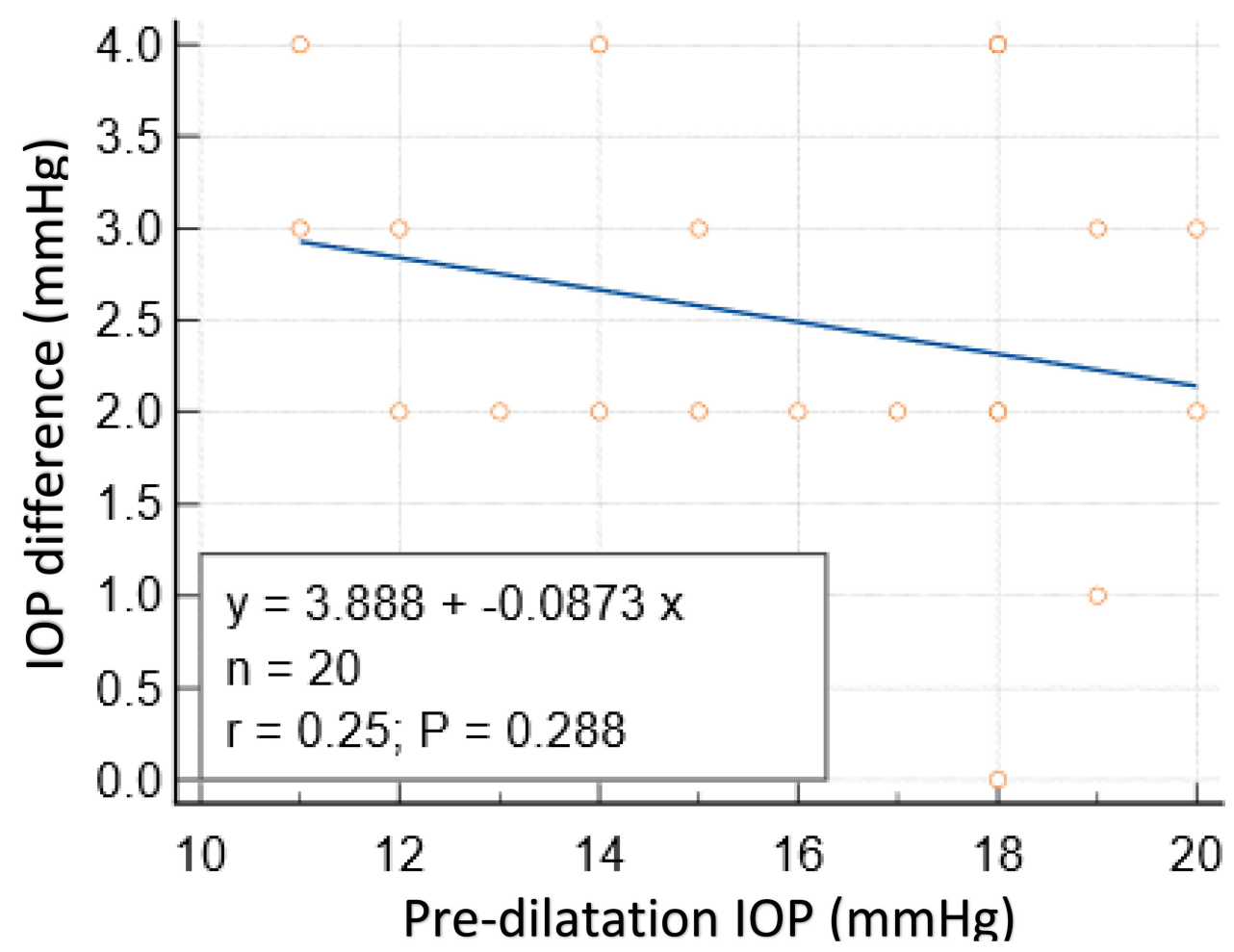

Figure 3 Linear regression analysis showing the correlation between the difference in IOP (pre-dilatation - post-dilatation) and the pre-dilatation IOP in group B. 
Moreover, in another study, the aqueous inflow and outflow rates through the trabecular meshwork were compared in responders and non-responders to cyclopentolate. The main difference found was the inflow rate, which decreased in non-responders but slightly increased in responders, while all patients had decreased aqueous humour outflow rate out of the trabecular meshwork. ${ }^{14}$ Similarly, in the current study group A, the reduction in the IOP following mydriasis could be explained by the enhanced inflow towards the trabecular meshwork caused by the response to mydriatic, ${ }^{14}$ which together with the enhanced aqueous outflow through the trabeculectomy can provide a hypothesis to explain the paradoxical reduction of IOP following mydriasis in trabeculectomy eyes.

Kim et al found that the anterior chamber depth increases with pupil dilatation, leading to a wider contact area between the trabecular meshwork and the aqueous. ${ }^{8}$ Moreover, in another study, ${ }^{15}$ the anterior chamber angle became wider with pupillary dilatation, possibly due to the posterior pull of the iris-lens diaphragm, leading to deepening of the anterior chamber. The widened anterior chamber angle created a larger surface area between the trabecular meshwork and the aqueous humour, eventually leading to an improvement in the aqueous outflow, which subsequently led to a drop in the IOP more than $6 \mathrm{~h}$ following pupillary dilatation after the initial IOP rise. Depending on the findings of the two above-mentioned studies, ${ }^{8,15}$ it could be assumed that pupil dilatation in the current study, resulted in widening of the anterior chamber angle, hence the trabeculectomy internal ostium, eventually leading to enhanced aqueous flow through the ostium, which could explain the drop in IOP following pupillary dilatation.

Quigley et $\mathrm{al}^{16}$ found that the iris thickness measured by optical coherence tomography (OCT) increases following pupil dilatation. This can cause angle crowding and impairment of aqueous drainage, resulting in raised IOP.

In the current study, group A eyes had peripheral iridectomy, which means that there is no iris tissue present opposite the trabeculectomy internal ostium, so the peripheral iris is unlikely to obstruct aqueous flow, even after dilating the pupil. Moreover, the peripheral iridectomy in these eyes diverts aqueous humour flow from the posterior chamber to the anterior chamber directly, bypassing its normal pathway through the pupil.

Furthermore, the trabecular meshwork obstruction with the iris pigments liberated following pupillary dilatation was supposed to be one of the mechanisms responsible for raising the IOP following mydriasis. ${ }^{4,17,18}$ As trabeculectomy bypasses the usual drainage of aqueous humour through the trabecular meshwork, and as the tiny iris pigments are unlikely to obstruct the relatively large trabeculectomy internal ostium, it is unlikely that iris pigment liberation will cause raised IOP in eyes with functioning trabeculectomy.

So, in summary, trabeculectomy bypasses the usual trabecular meshwork resistance. In addition, peripheral iridectomy opposite the trabeculectomy internal ostium makes it unlikely for the iris tissue to obstruct the ostium. Moreover, iris pigments liberated following mydriasis are unlikely to obstruct the ostium.

In group A, the IOP level was unchanged following mydriasis in 4 eyes, these 4 eyes $(21.1 \%)$ had IOP $12 \mathrm{mmHg}$ or less, however, the other 15 eyes (78.9\%) showed a drop of the IOP following pupillary dilatation, no eyes had IOP rise following mydriasis. On the other hand, the IOP rose in $95 \%$ of group B eyes following mydriasis, only one eye (5\%) had the same IOP post-dilatation, no eyes had IOP drop following mydriasis, which is comparable to the usual effect of mydriasis on IOP shown in previous studies. ${ }^{3,8-12}$ There was a strong negative correlation between the pre-dilatation IOP and the difference (pre-dilatation - post-dilatation), which means that higher pre-dilatation IOP was associated with higher negative (broader) difference. This means that the drop in IOP following pupillary dilatation was higher in group A eyes with higher IOP values than in eyes with less IOP. This could be attributed to the fact that eyes with lower IOP values following trabeculectomy already have excellent drainage through the trabeculectomy site which is less likely to be enhanced by minor factors such as pupillary dilatation. Although the small sample regression analysis could be associated with higher false positive and false negative results, a study found that this mainly occurred at samples smaller than $<8$, but data shape was clearly identified at $\mathrm{N} \geq 8$. $^{19}$

Limitations of this study included the small number of eyes included. Moreover, the IOP measurement by the applanation tonometer could carry more bias compared to other techniques as non-contact tonometer, or tonopen.

In clinical practice, this novel finding could help to predict the actual IOP in a non-dilated pupil in case a patient presents to the clinic with already dilated pupil. Furthermore, this work could represent a possible novel sign for assessment of bleb function with further studies. 


\section{Précis}

In this study, there was a drop of intraocular pressure after mydriasis in eyes with functioning trabeculectomy blebs, in contrast to the usual effect of mydriasis on intraocular pressure as in the control group.

\section{Acknowledgments}

I would like to acknowledge Dr Hesham Mohamed, who assisted me in performing the statistical analysis in this work. This article was not previously published or under publication or presented anywhere.

\section{Funding}

This work did not receive any financial grants or funds.

\section{Disclosure}

The author reports no conflicts of interest for this work.

\section{References}

1. Dailymed. Mydriacil - Tropicamide Solution/Drops. USA: NIH; 2019. Available from: dailymed.nlm.nih.gov. Accessed January 19, 2022.

2. Adediji AK, Adio AO, Fiebai B. Effects of diagnostic mydriasis with tropicamide and phenylephrine on intraocular pressure. J Ophthalmic Clin Res. 2019;6(1):49.

3. Velasco Cabrera J, Eiroa Mozos P, Garcia Sanchez J, Bermudez Rodriguez F. Changes in intraocular pressure due to cycloplegia. CLAO J. 1998;24 (2):111-114

4. Kristensen P. Mydriasis-induced pigment liberation in the anterior chamber associated with acute rise in intraocular pressure in open-angle glaucoma. Acta Ophthalmol. 1965;43(5):714-724. doi:10.1111/j.1755-3768.1965.tb00343.x

5. Harris LS, Galin MA, Mittag TW. Cycloplegic provocative testing after topical administration of steroids. Arch Ophthalmol. 1971;86(1):12-14. doi:10.1001/archopht.1971.01000010014003

6. One network: gonioscopic grading systems [Internet] US: American Academy of Ophthalmology;, 2017. Available from:: www.aao.org. Accessed January 19, 2022.

7. Cantor LB, Mantravadi A, WuDunn D, Swamynathan K, Cortes A. Morphologic classification of filtering blebs after glaucoma filtration surgery: the indiana bleb appearance grading scale. J. Glaucoma. 2003;12:266-271. doi:10.1097/00061198-200306000-00015

8. Kim JM, Park KH, Han SY, et al. Changes in intraocular pressure after pharmacologic pupil dilation. BMC Ophthalmol. 2012;12:15-53. doi:10.1186/1471-2415-12-15

9. Shaw BR, Lewis RA. Intraocular pressure elevation after pupillary dilation in open angle glaucoma. Arch Ophthalmol. 1986;104(8):1185-1188. doi:10.1001/archopht.1986.01050200091057

10. Hancox J, Murdoch I, Parmar D. Changes in intraocular pressure following diagnostic mydriasis with cyclopentolate 1\%. Eye (Lond). 2002;16:562-566. doi:10.1038/sj.eye.6700146

11. Archini G, Babighian S, Tosi R, Perfetti S, Bonomi L. Comparative study of the effects of $2 \%$ ibopamine, $10 \%$ phenylephrine and $1 \%$ tropicamide on the anterior segment. Invest Ophthalmol Vis Sci. 2003;44:281-289. doi:10.1167/iovs.02-0221

12. Siam GA, Monteiro de Barros DS, Gheith ME, et al. The amount of intraocular pressure rise during pharmacological pupillary dilatation is an indicator of the likelihood of future progression of glaucoma. Br J Ophthalmol. 2007;91:1170-1172. doi:10.1136/bjo.2007.116855

13. Foss AJE, Shah P. Glaucoma surgery. In: Essential Ophthalmic Surgery. 1st ed. Oxford: Butterworth Heinemann; 2001:209-225.

14. Valle O. Effect of cyclopentolate on the aqueous dynamics in incipient or suspected open-angle glaucoma. Acta Ophthalmol Suppl. 1974;123:52-60.

15. Kim KS, Kim JM, Park KH, Choi CY, Chang HR. The effect of cataract surgery on diurnal intraocular pressure fluctuation. J Glaucoma. 2009;18 (5):399-402. doi:10.1097/IJG.0b013e3181879e89

16. Quigley HA, Silver DM, Friedman DS, et al. Iris cross-sectional area decreases with pupil dilation and its dynamic behavior is a risk factor in angle closure. J Glaucoma. 2009;18:173-179. doi:10.1097/IJG.0b013e31818624ce

17. Kristensen P. Pigment liberation test in open-angle glaucoma. Acta Ophthalmol (Copenh). 1968;46(3):586-599. doi:10.1111/j.1755-3768.1968. tb02855.x

18. Valle O. The cyclopentolate provocative test in suspected or untreated open-angle glaucoma. V. Statistical analysis of 431 eyes. Acta Ophthalmol (Copenh). 1976;54(6):791-803. doi:10.1111/j.1755-3768.1976.tb01800.x

19. Jenkins DG, Quintana-Ascencio PF. A solution to minimum sample size for regressions. PLoS One. 2020;15(2):e0229345. doi:10.1371/journal. pone. 0229345 


\section{Publish your work in this journal}

Clinical Ophthalmology is an international, peer-reviewed journal covering all subspecialties within ophthalmology. Key topics include: Optometry; Visual science; Pharmacology and drug therapy in eye diseases; Basic Sciences; Primary and Secondary eye care; Patient Safety and Quality of Care Improvements. This journal is indexed on PubMed Central and CAS, and is the official journal of The Society of Clinical Ophthalmology (SCO). The manuscript management system is completely online and includes a very quick and fair peer-review system, which is all easy to use. Visit http://www. dovepress.com/testimonials.php to read real quotes from published authors.

Submit your manuscript here: https://www.dovepress.com/clinical-ophthalmology-journal 